Musées, Patrimoine et Culture scientifiques et techniques

$167 \mid 2016$

septembre-octobre 2016

\title{
Le patrimoine universitaire toulousain passé au crible
}

\section{Anne-Claire Jolivet et Marie-Charlotte Mazens}

\section{OpenEdition \\ Journals}

Édition électronique

URL : http://journals.openedition.org/ocim/1694

DOI : $10.4000 /$ ocim. 1694

ISSN : 2108-646X

Éditeur

OCIM

Édition imprimée

Date de publication : 1 septembre 2016

Pagination : patrimoine universitaire

ISSN : 0994-1908

\section{Référence électronique}

Anne-Claire Jolivet et Marie-Charlotte Mazens, « Le patrimoine universitaire toulousain passé au crible ", La Lettre de l'OCIM [En ligne], 167 | 2016, mis en ligne le 01 septembre 2017, consulté le 01 mai 2019. URL : http://journals.openedition.org/ocim/1694; DOI : 10.4000/ocim.1694

Ce document a été généré automatiquement le 1 mai 2019.

Tous droits réservés 


\title{
Le patrimoine universitaire toulousain passé au crible
}

\author{
Anne-Claire Jolivet et Marie-Charlotte Mazens
}

Deux robots d'explorations planétaires sur leur terrain d'expérimentation.

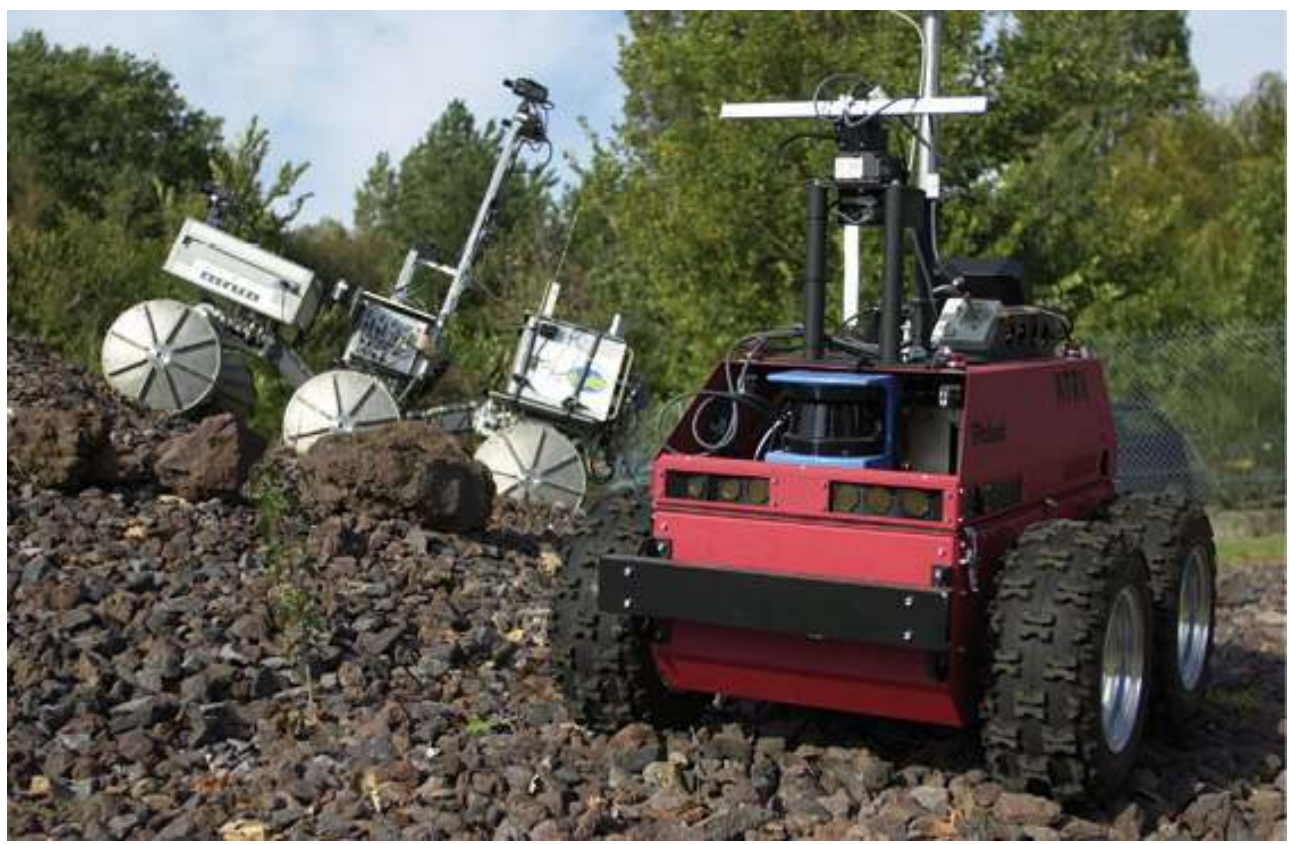

(C) Laboratoire d'Analyse et d'Architecture des Systèmes, CNRS

Outre leur visée universelle, les mondes scientifiques sont ancrés dans un territoire géographique, politique et culturel. Le contexte socio-économique influe sur les partenariats et les orientations thématiques; les politiques nationales, régionales voire municipales soutiennent plus ou moins généreusement la mise en place d'équipements, de bâtiments ou directement de programmes de recherche; les interactions avec la cité permettent la construction de projets culturels, de stratégies de communications et/ou de pratiques mémorielles. En 2016, l'université se doit d'être visible et optimisée : gérer 
son image et ses ressources à l'échelle locale, nationale et internationale représente de nouvelles priorités impulsées notamment par la LRU, les IDEX et la mise en place des COMUE. Dans ce contexte, le patrimoine scientifique peut sembler utile pour s'affirmer d'un point de vue historique et culturel ; par ailleurs, conserver, documenter, valoriser coûte et prend du temps.

2 Si le musée propose un cadre modèle de conservation, d'études et de monstration des traces du passé, toutes les universités françaises ne se sont pas, pour des raisons économiques notamment, orientées vers la création de ce type d'infrastructures. Et, même affranchis des limites pécuniaires, leurs personnels (administratifs, chercheurs, enseignants) ne souhaitent pas toujours voir se développer une politique patrimoniale classique, peu adaptée aux spécificités du patrimoine universitaire. En effet, la constitution de collections universitaires s'inscrit dans une dynamique propre au milieu scientifique. Elles ne sont pas issues d'un programme d'acquisition tel que ceux développés par les musées afin d'enrichir une collection préexistante, mais sont avant tout des collections pédagogiques, et des collections utilisées pour la recherche.

À Toulouse, tout a commencé par le patrimoine des bibliothèques : planche du traité complet de la médecine des chevaux de Philippe-Étienne Lafosse.

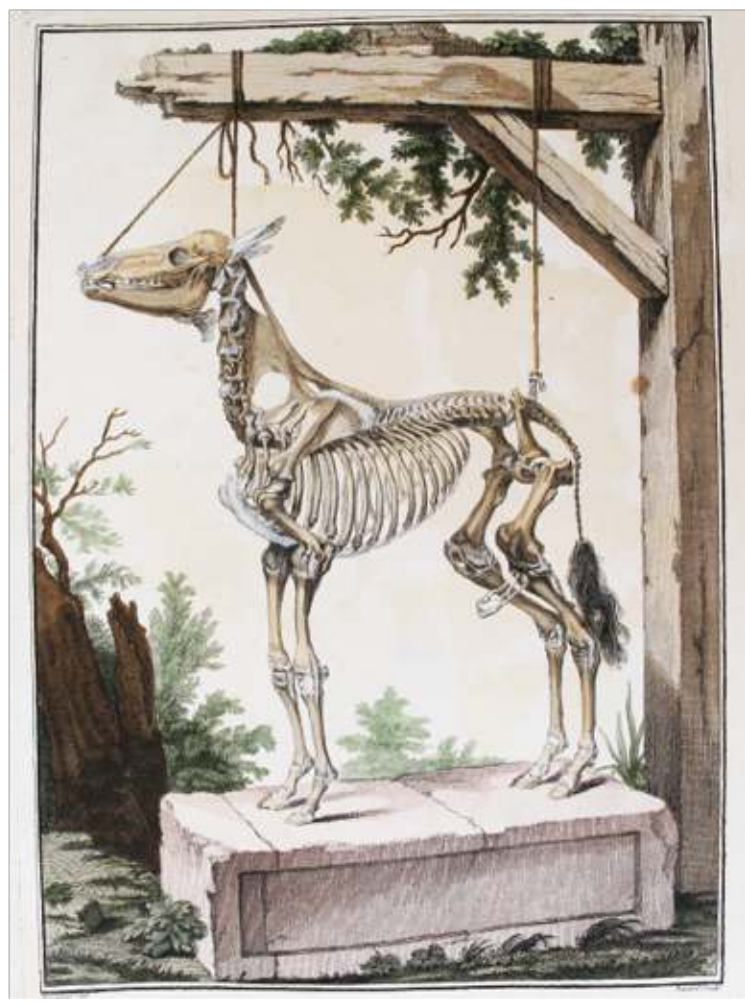

(c) Sarah Martin

Dans le monde pluridisciplinaire de l'université, parlons-nous tous du même patrimoine ? La polysémie des termes "patrimoine", "patrimoine scientifique", "patrimoine universitaire" ou "collections", de même que l'affect projeté sur ces matériels qui ont accompagné la carrière de nombres d'acteurs, ne rendent pas toujours les séances collectives de travail fluides et efficaces. Ainsi, la subjectivité inhérente et les acceptions disciplinaires de ces notions nécessitent de décrire tous les ensembles patrimoniaux ressentis comme tels par la communauté mobilisée. À défaut d'apporter notre définition 
du patrimoine universitaire, cette étude décline les patrimoines qui font sens pour les uns ou les autres membres de l'université Fédérale Toulouse Midi-Pyrénées (UFTMiP).

\section{Les prémices du projet dans un contexte universitaire toulousain en mouvement}

Depuis les années 1990, et La Lettre de l'OCIM s'en est souvent fait l'écho, de multiples universités françaises et européennes s'emparent de leurs patrimoines ${ }^{1}$. À Toulouse, cette démarche se formalise avec le patrimoine des bibliothèques et s'intensifie encore au tournant des années 2000 donnant jour à de nombreuses initiatives². À l'automne 2013, les établissements membres de l'UFTMiP validaient la formation d'un groupe de travail transversal, composé de spécialistes œuvrant déjà au sein des établissements à titre professionnel ou bénévole, reconnus pour leur connaissance de l'histoire de leur discipline, d'une collection ou de leur institution. Ce groupe de travail "Patrimoine culturel universitaire" est placé sous la houlette avisée du regretté professeur Georges Larrouy ${ }^{3}$, avec l'assistance de la composante Diffusion de la Culture des Sciences et des Techniques (DCST). Se réunissant tous les 3 mois, plus de 30 personnes ont piloté une étude permettant de mieux connaitre l'étendue du patrimoine universitaire toulousain. Elle s'inscrit dans l'une des trois orientations de l'axe "Diffusion des savoirs" de l'IDEX,' consacrée à "la valorisation du patrimoine culturel universitaire".

5 Nous avons veillé à ce que la diversité des ensembles patrimoniaux repérés soit représentée et nous nous sommes appuyés sur les précieuses compétences des chercheurs en sciences humaines et sociales directement concernés par notre sujet d'étude (histoire, archéologie, anthropologie, sociologie) ainsi que des responsables de musées et de centres de culture scientifique. 2014 était l'année de discussion des statuts et du règlement intérieur de notre jeune entité fédérale ${ }^{5}$; le climat politique interne n'étant pas toujours apaisé, il a été pris grand soin de faire valider ces référents-experts par les directions de chaque établissement concerné. Dans ce contexte mouvementé, qui agite régulièrement une institution à géométrie variable entre cloisonnement et réunification ${ }^{6}$, les problématiques liées à la sauvegarde, la conservation et la valorisation du patrimoine universitaire sont trop souvent considérées comme dérisoires ou secondaires, ou alors instrumentalisées comme recours identitaire et pré-carré de certains établissements.

\section{La mission et ses objectifs}

6 Partant de cette perception empirique que chaque établissement de l'université de Toulouse détient un patrimoine historique, scientifique, architectural et artistique méconnu ou peu exploité, l'objectif initial de cette étude était de proposer à ses membres un état des lieux de ces patrimoines. Puis, à partir des données de cet "inventaire des inventaires", établir des priorités d'actions réalistes en fonction des moyens potentiellement allouables.

7 En s'immergeant dans ce recensement, il est rapidement apparu essentiel de profiter de cette enquête pour, d'une part, poser les bases d'un outil de gestion interrogeant les usages et les rôles actuels de ces patrimoines et, d'autre part, réunir les éléments permettant une future valorisation grand public de chaque collection (historique, auteur, motivation de collecte, évolution, éléments remarquables, quelques illustrations de 
qualité...). Pour se laisser le temps de satisfaire ces deux objectifs complémentaires, il a fallu limiter le secteur d'investigation aux seuls établissements toulousains afin de ne pas se disperser sur la grande région Midi-Pyrénées, dans le souhait d'étendre ultérieurement ce recueil de données à l'ensemble des sites?

8 L'objectif stratégique de cette étude, s'oriente alors vers la conception d'un outil de prise de conscience et de promotion à destination des instances dirigeantes des établissements de recherche et d'enseignement supérieurs de l'UFTMiP. Il permet de démystifier beaucoup d'a priori en jugeant de l'état et de l'importance de ces patrimoines. En effet, par trop méconnus, ces ensembles patrimoniaux ont pu être perçus comme oubliés ou cachés, incommensurables et insondables, sans aucune ou ayant une grande valeur marchande, inutiles ou terriblement en péril. Par cet état des lieux, nous espérons avoir dissipé fantasmes et angoisses agitant les consciences à la seule évocation de ces patrimoines.

\section{Des grilles analytiques à l'épreuve de la disparité des collections}

9 Après avoir identifié les ensembles patrimoniaux et les personnes qui les connaissent le mieux, il a fallu se confronter à la disparité de ces ensembles. Mais comment révéler à la fois l'objet, la collection, l'auteur et l'acteur d'un patrimoine scientifique? Une grille analytique unique a été conçue pour chaque ensemble patrimonial identifié. Leurs composantes partagent des critères communs connus ou supposés (nature, identité du collecteur, période ou zone de collecte, localisation... et bien d'autres encore). Pour répondre à un besoin de standardisation nécessaire à l'exercice et de lisibilité des données, cette grille contient plusieurs blocs d'informations liés aux quatre étapes incontournables de la mise en patrimoine. Dans la vie d'un fonds patrimonial, ces étapes se succèdent, s'interpénètrent et interagissent les unes avec les autres :

10 - l'identification (la dénomination, le descriptif, l'origine, l'historique, les contributeurs, les éléments remarquables du fonds, la localisation, les organismes détenteurs, les droits liés à la propriété intellectuelle, et des liens vers d'autres ensembles connexes);

11 - la conservation (l'inventaire, la numérisation, les lieux d'exposition ou d'entreposage, l'accessibilité, la régie et la sécurité des biens et des personnes), le cadre de la collection (état sanitaire, accessibilité, moyens dédiés) et la vie du fonds (fréquentation, objectifs, collaborations, besoins et urgences et axes de recherche);

12 - la documentation (les documents associés, l'illustration, la bibliographie et les personnes ressources susceptibles d'apporter de plus amples informations);

13 - la valorisation (publications, visites, expositions, pages Internet...). 
Grille de lecture appliquée pour l'étude à chaque ensemble patrimonial identifié.

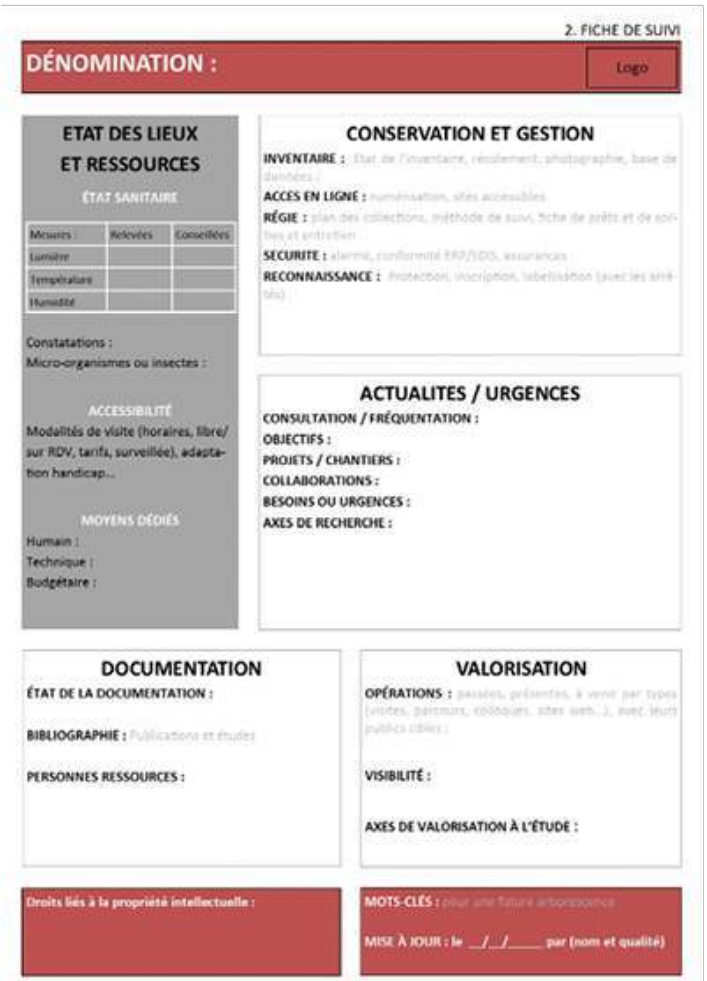

(c) Université de Toulouse

14 Cette enquête a permis de réaliser un état des connaissances en soulevant de nombreuses questions dont certaines restent encore sans réponse. Nous avons souhaité restituer ces carences par la mention "N.C." (pour "non communiqué") sur les grilles analytiques pour que le lecteur connaisse les compléments d'informations à apporter ultérieurement. Ces grilles restent évolutives au gré des découvertes sur la collection. Ainsi, plus qu'un instantané de la situation 2014-2015, cet outil se veut exploitable par la suite. Facile à mettre à jour, la méthodologie d'étude définie permet d'adjoindre à cet "inventaire des inventaires" toute nouvelle fiche concernant un ensemble patrimonial non encore étudié à ce jour et de sortir du périmètre toulousain pour gagner progressivement l'ensemble des établissements de l'UFTMiP. 
Le générateur de haute tension à l'intérieur de la "Boule" de l'ancien laboratoire d'optique électronique (aujourd'hui CEMES)

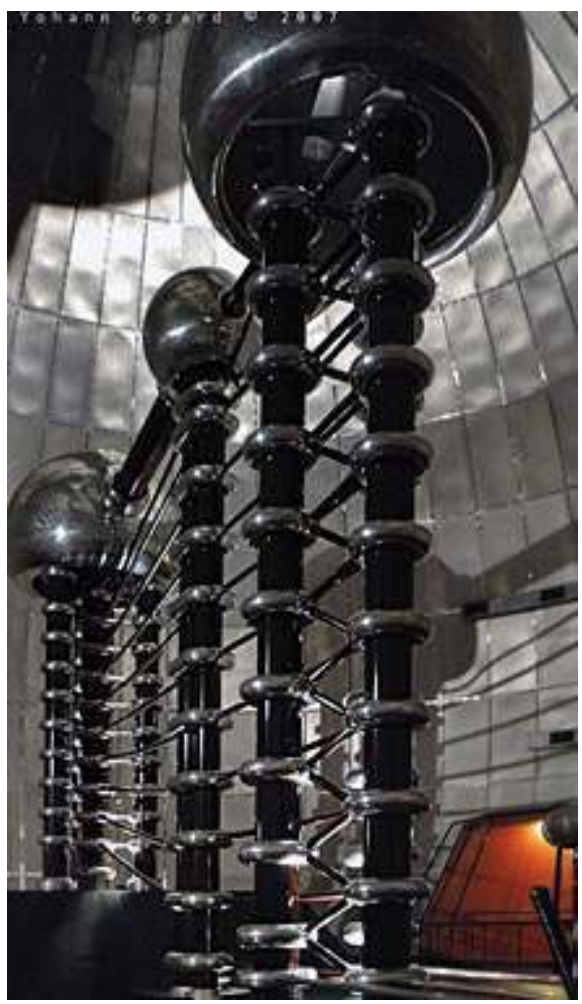

(c) Yohann Gozard 


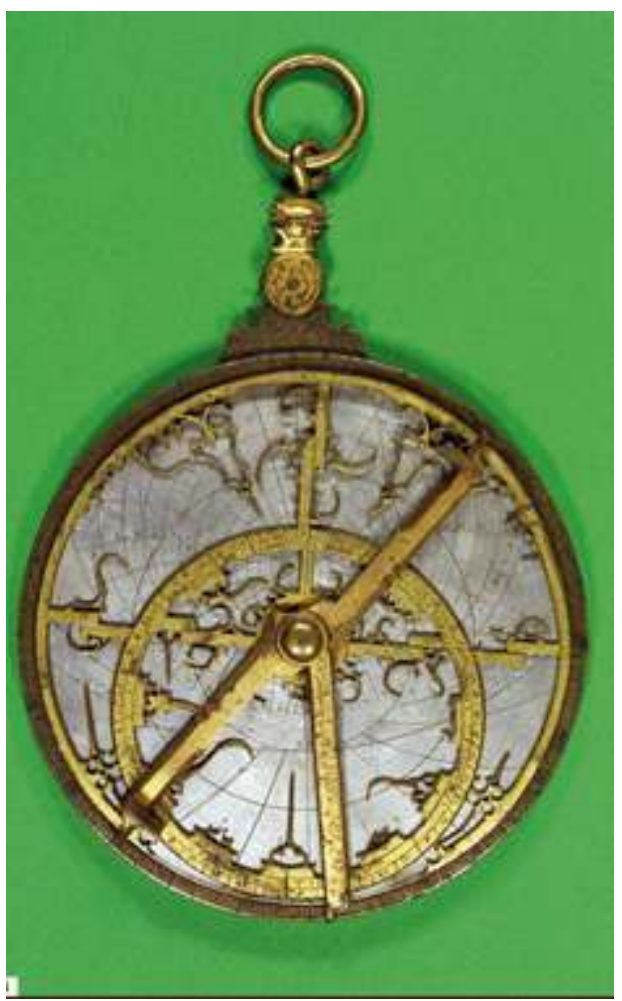

(c) Observatoire Midi-Pyrénées, en dépôt au musée Paul Dupuy

\section{L'importance, l'hétérogénéité et la difficulté de prise en charge du patrimoine universitaire toulousain}

La communauté universitaire toulousaine détient aujourd'hui une multitude de fonds issus principalement des évolutions et spécialités de son activité propre : la recherche et l'enseignement. Toutefois, ils peuvent également provenir de son administration, ses pratiques, ses lieux et ses usages ou encore résulter de donations (parfois sans rapport avec l'activité universitaire), léguées par des institutions ou des personnalités qui ont reconnu en l'université une légitimité pour en assurer la permanence, l'étude et la transmission. Ainsi, la longue histoire de l'université de Toulouse, fondée en 1229, et sa pluridisciplinarité nous font les héritiers d'un patrimoine scientifique considérable mais également un précieux patrimoine architectural, artistique et écrit dont ce recensement nous dévoile la richesse, la typicité et l'unicité. Il rassemble des collections zoologiques, paléontologiques, minéralogiques, cartographiques, anatomiques, odontologiques, anthropologiques, astronomiques, un jardin botanique et un arboretum, des herbiers, des fonds photographiques, des instruments scientifiques anciens et contemporains, des supports pédagogiques... mais aussi (hors du strict domaine scientifique) un important patrimoine écrit, des archives (papier, numériques, audiovisuelles...), des œuvres d'art, des bâtiments et des complexes architecturaux et paysagers. Ce patrimoine culturel compte parmi les plus importants des universités françaises. À titre d'exemple, avec leurs 113340 volumes signalés dans cette étude, les établissements toulousains possèdent, après les bibliothèques parisiennes, le deuxième fonds de livres anciens de France. 
16 Il est également reconnu par toutes les institutions culturelles qui créditent et soutiennent nos actions en sa faveur, classent ou inscrivent les éléments les plus remarquables. Il faut ici souligner le rôle de premier plan de nos partenaires institutionnels qui conservent une partie de notre patrimoine et nous apportent ponctuellement leur expertise. Les archives départementales, les archives municipales ou encore les musées de Toulouse veillent sur des collections qui n'auraient pu trouver pareil soin au sein de nos établissements qui ne disposent que très rarement de structures de conservation dédiées (et le plus souvent non conformes aux normes des Établissements Recevant du Public-ERP).

Cette masse et cette diversité ne nuisent évidemment pas à la richesse du patrimoine universitaire mais troublent sa visibilité et complexifient sa prise en charge. Il faut aujourd'hui investiguer plus en profondeur ces différents ensembles patrimoniaux et s'interroger sur le statut de nos collections et les processus de patrimonialisation à l'œuvre. La multiplicité des formes et les impressionnants volumes rendent fastidieuses les campagnes d'inventaire. C'est pourtant la première action à mener pour approfondir notre connaissance des fonds et ainsi pouvoir identifier et faire la part entre les différents statuts de collections. Se trouvent aujourd'hui pêle-mêle des pièces pouvant aussi bien appartenir à des notions toujours en quête de consensus telles que les "collection de référence" (qui devraient être conservées dans leur globalité comme les herbiers, les droguiers...), les "collections d'étude" (matériel perçu comme source ou moyen de la recherche, susceptible d'être encore utilisé et peut-être dégradé ou détruit), des "collections pédagogiques" (matériel de démonstration soutenant une transmission des savoirs, dont la manipulation peut être à la longue contraire à sa préservation), ou des "collections strictement patrimoniales" (matériel considéré comme devant être préservé devant tout autre usage comme les types et figurés)... Ces statuts liés à la destination des pièces des collections amènent directement les questions de publics, de protections (notamment juridique) et de modes de gestion adaptés et spécifiques à chacun d'entre eux. A contrario, la segmentation des fonds par leur nature intrinsèque (archives, livres, bâtiments, instruments, collections, bases de données...) crée une superposition de cadres conceptuels, administratifs et législatifs contraire à la nature transversale des fonds universitaires et en inadéquation avec les moyens dont dispose l'institution.

\section{Le rapport, un outil de travail et de séduction... mais aussi un exercice d'équilibrisme politique}

Ce rapport, initialement prévu comme une restitution synthétique, presque administrative, des données et des conclusions de l'étude sur le patrimoine culturel universitaire pour les tutelles dirigeantes des établissements, est progressivement devenu un outil de valorisation et de séduction à destination de la communauté universitaire toulousaine. Gardant une visée de diffusion interne, il a néanmoins été ouvert à nos partenaires institutionnels culturels et politiques comme un catalogue de présentation de nos collections et comme un document établissant des perspectives d'actions pouvant être réalisées en coopération. La mise en page de ces informations s'est organisée en deux volets : un volet "tout public" pour une présentation aisée et globale du fonds, et un volet "professionnel" utile à sa bonne gestion. Les données sensibles relatives à la sécurité des biens ont été retirées. 
Collections de paléontologie disposées après le déménagement dans les meubles d'origine.

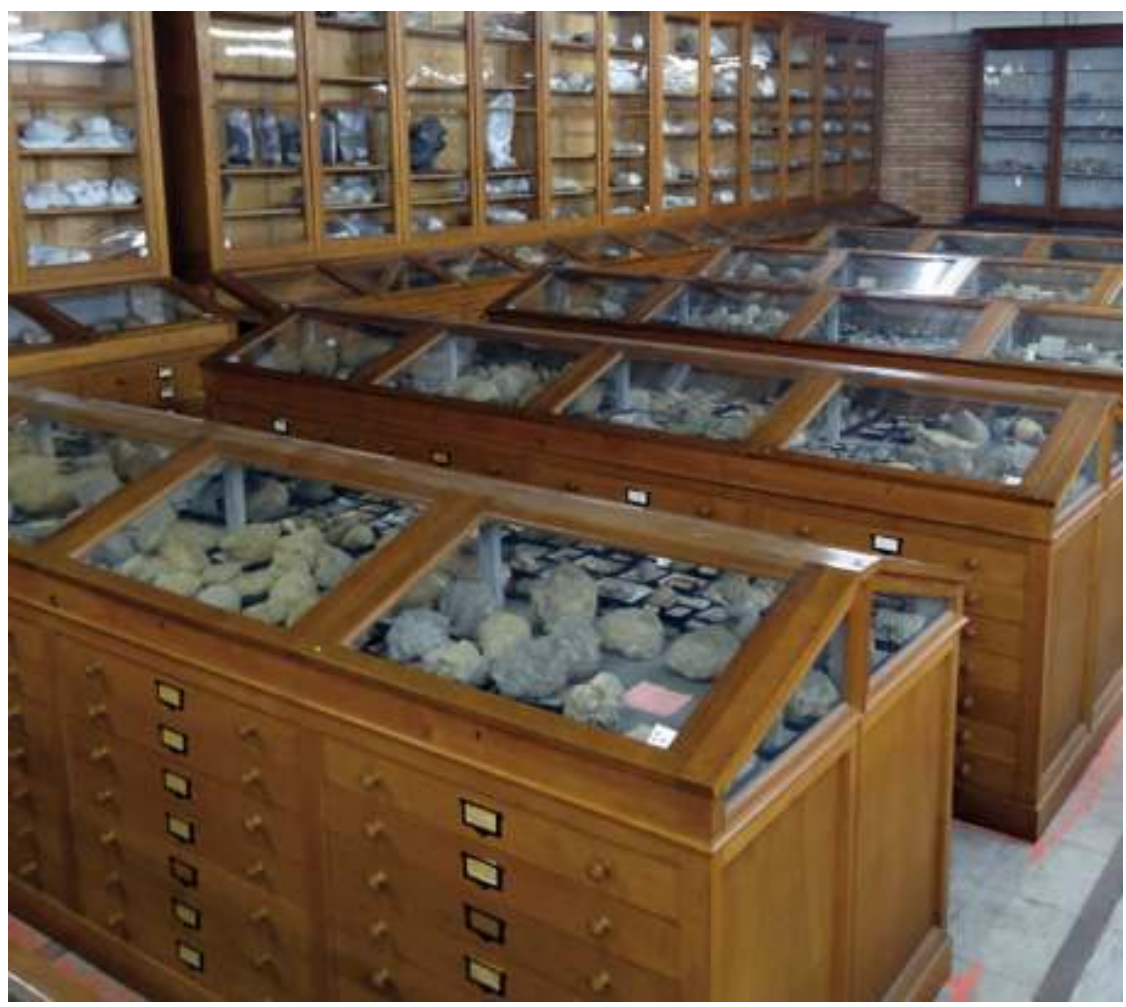

(c) $\mathrm{DR}$

19 Cependant, une autre évolution majeure a été générée par les dernières restitutions devant les instances politiques pourtant informées régulièrement des avancées de notre travail et mandataires de l'étude sur l'ensemble des patrimoines universitaires (bâti, écrit, archivistique, artistique et scientifique). Les perspectives de publication ont provoqué une réaction inattendue à ce stade. Alléguant de liens entre des vols et un précédent inventaire du patrimoine artistique, certains officiels ont exigé le retrait de ces pages et la partie sur le patrimoine artistique de l'ensemble des établissements a fait les frais du climat politique et de rapports de forces complètement étrangers à la question patrimoniale. Nous regrettons que le patrimoine artistique, soit absent de l'étude publiée à ce jour. Nous espérons qu'une prochaine version permettra d'éditer ce patrimoine hérité ou contemporain, le plus souvent lié aux personnalités et disciplines enseignées ou inspiré par la dynamique du progrès et de l'innovation... Il s'en est fallu de peu que le patrimoine bâti ne disparaisse également du rapport, par crainte d'une hypothétique ingérence sur ces biens administrés et valorisés par les services immobiliers des établissements qui gèrent quotidiennement leur exploitation. 


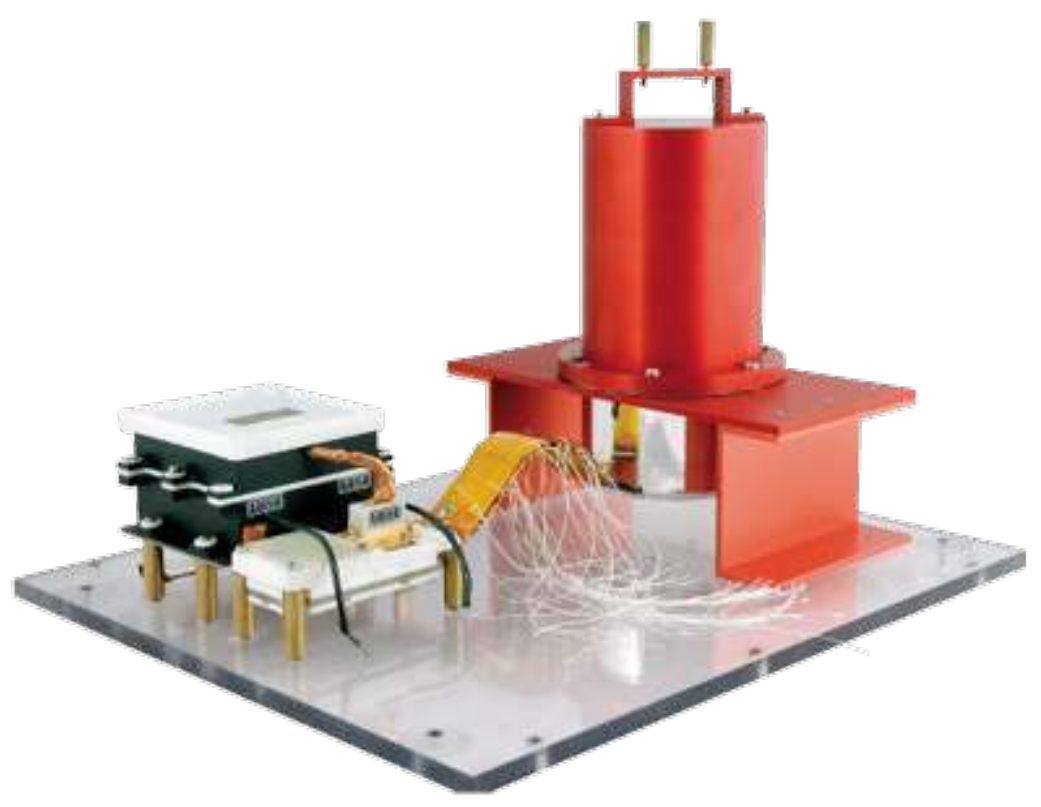

(c) PATSTEC, Institut de Recherche en Astrophysique et Planétologie

20 Le rapport présente finalement les patrimoines scientifiques au sens large, et au pluriel, qui représentent tout de même la plus grande partie des ensembles patrimoniaux des établissements de l'UFTMiP. En effet, la quasi-totalité du patrimoine de l'université, lieu de sciences et de savoirs, pourrait être entendue comme "patrimoine scientifique". Certain bâtiments sont des instruments monumentaux dédiés à la recherche expérimentale; les archives, comme le patrimoine écrit, ne sont autres que les vestiges textuels ou iconographiques des activités de recherche et d'enseignement. L'inventaire publié regroupe ainsi :

21 - le patrimoine archivistique (archives administratives ou scientifiques) : immense et encore peu investigué, il reste méconnu et se trouve en situation d'urgence car il ne fait que trop rarement l'objet d'un suivi professionnel.

22 - le patrimoine des bibliothèques universitaires : il présente les documents considérés comme "rares, anciens ou précieux" pour nous affranchir des débats de limites de datations et ses collections sont identifiées, protégées et mises à disposition des publics. Ce constat très positif est le résultat d'une prise en charge pérenne, consultée et mutualisée, patiemment orchestrée sur les vingt dernières années par un personnel qualifié. Un exemple à suivre pour les autres typologies du patrimoine universitaire toulousain.

23 - les collections de références et instrumentales : les collections de références rassemblent des matériels collectés, acquis ou légués, liés essentiellement aux sciences naturelles et aux sciences médicales. Malgré d'impressionnants volumes, elles sont progressivement investies et investiguées par les établissements. Les collections instrumentales se composent des matériels obsolètes utilisés ou produits par la recherche et l'enseignement des sciences et des techniques. La mission PATSTEC, active depuis 2004, s'appuie sur la mission nationale pilotée par le musée des Arts et Métiers et l'aide de nombreux spécialistes pour sauvegarder ce patrimoine instrumental en constante fabrication dans les laboratoires. 
- les sites historiques antérieurs à 1969 : ce dernier patrimoine, le bâti, ayant été réintroduit en fin de rapport comme appendice dessinant le cadre de l'étude, soutient la présentation historique de l'institution universitaire et révèle la dimension archéologique du site avec son mille-feuille de strates historiques. C'est avec lui, l'évolution de la ville de Toulouse qui se dévoile en partie.

Le laboratoire de chimie de Paul Sabatier au début du XXe siècle

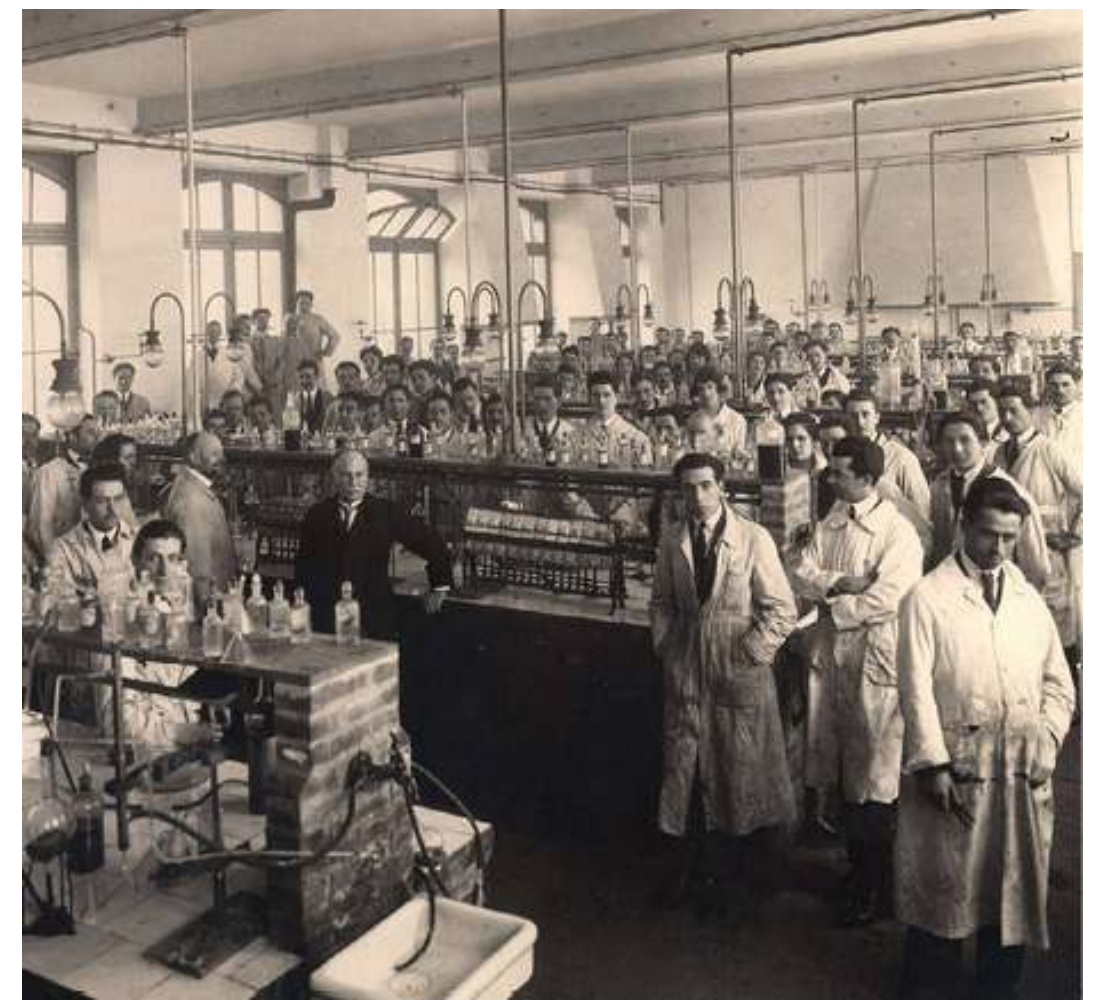

(c) DR

Alors que nous visions initialement une présentation par établissement pour en écrire l'histoire et saluer le chemin parcouru ainsi que l'implication, les efforts et les initiatives de leur personnel, nous avons prudemment opté pour une présentation par type de patrimoine. Ainsi, nous évitons de pointer des différences de pratiques ou d'investissement entre les établissements et nous fédérons la communauté par une présentation décloisonnée des traces de son histoire. À l'intérieur de chacune de ces typologies, les grilles analytiques ont été retravaillées et illustrées pour un plus grand plaisir de lecture. Ces fiches sont précédées par une rubrique intitulée "De quoi s'agit-il ?" pour cadrer le domaine d'étude, puis d'une "Présentation transversale", qui permet déjà d'écrire à gros traits la singularité des sciences toulousaines. Elles sont suivies par un "État des lieux" centré sur l'identification, la conservation, la documentation et la valorisation qui permet d'amener des "Recommandations" spécifiques à ce type de patrimoine, avant d'en établir d'autres en vue d'une possible politique patrimoniale plus générale en fin de rapport. 


\section{Réinvestir notre héritage culturel}

L'étude révèle une question essentielle : existe-t-il, en 2016, un consensus pour construire une identité commune de site et mettre en valeur une histoire prestigieuse partagée ? Si la réponse est "oui", il est primordial d'avancer pas à pas en organisant une concertation de site pour l'élaboration d'une politique patrimoniale graduelle qui capitalise les acquis et écoute les différents rythmes et moyens de chaque établissement. Si la réponse est "pas tout de suite", il reste possible de progresser durablement au niveau de chaque établissement en renforçant, notamment, la présence du patrimoine dans les activités de recherche et d'enseignement et en soutenant les personnels qui s'en préoccupent.

Nous avons priorisé schématiquement les propositions d'actions, à mener à l'échelle d'un établissement ou du site, en les organisant par axes et en identifiant celles possibles quasi immédiatement à moindre coût et celles nécessitant plus de temps ou de moyens. Elles s'organisent en cinq axes :

- L'identification des acteurs impliqués, leur coordination et la pérennité des données

- L'approfondissement de la connaissance des ensembles patrimoniaux

- La mise en scène et en récit de ces patrimoines pour le rayonnement de l'université

- La place du patrimoine dans les missions de recherche et d'enseignement

- La reconnaissance institutionnelle du patrimoine universitaire

Ces axes de travail pourraient constituer une vraie avancée patrimoniale. Ils permettraient d'asseoir les collections sur une base solide et d'esquisser les bases d'une politique patrimoniale progressive et concertée à l'échelle de l'établissement et/ou du site avec l'aide bienveillante de nos partenaires institutionnels culturels (archives, musées, DRAC) et des collectivités territoriales.

4 Les établissements de l'UFTMiP sont pour l'heure dans un entre deux. S'ils souhaitent désormais conserver leur patrimoine, il leur reste à s'approprier la pluralité de son potentiel, à définir son rôle et à intensifier l'approfondissement des connaissances de leurs collections, en établissant sur le temps long, un schéma opérationnel définissant ce qu'ils veulent en faire et à se doter progressivement des moyens d'une prise en charge minimale. C'est dans cet objectif que nous avons rédigé en 2015 le rapport de l'étude sur le patrimoine universitaire (disponible pour tous, en version numérique, sur le site de l'UFTMiP) comme un outil d'aide à la décision et présenter les recommandations du groupe de travail ${ }^{8}$. Suite à l'annonce de l'arrêt de l'IDEX, la communauté universitaire toulousaine doit capitaliser ses acquis tout en innovant sur les manières d'être ensemble ; les négociations promettent d'être intenses, le patrimoine scientifique constituera-t-il un enjeu qui méritera débat à l'échelle du site? 


\section{NOTES}

1. Ferriot, D et Lourenço, M. De l'utilité des musées et collections des universités, La Lettre de l'OCIM, $\mathrm{n}^{\circ}$ 93, pp. 4-16; Soubiran, S. Patrimoine des universités et médiation scientifique, La Lettre de l'OCIM, n 109, pp. 33-41; Lourenço M.-C., Soubiran, S., Wittje, R., Talas, S. et Bremer, T. Initiatives européennes et patrimoine universitaire, La Lettre de l'OCIM, $\mathrm{n}^{\circ} 123$, pp. 5-14 ; Soubiran, S. et Maison-Soulard, L. Musées et collections universitaires : nouveaux enjeux, nouvel élan, La Lettre de l'OCIM, $\mathrm{n}^{\circ} 129$, pp. 5-6; Soubiran, S. Patrimoine des universités et médiation culturelle des sciences, La Lettre de l'OCIM, $\mathrm{n}^{\circ}$ 164, pp. 33-37.

2. Nous citerons ici : le Service du livre ancien du Service Inter-établissements de Coopération Documentaire (SICD) qui conserve, numérise et valorise le patrimoine des bibliothèques universitaires du site depuis 1995, la commission Patrimoine de l'Observatoire Midi-Pyrénées, active depuis 1992 et la mission PATSTEC mise en place dès 2004 en Midi-Pyrénées et le colloque du réseau UNIVERSEUM en 2009 (voir à ce sujet le $n^{\circ} 129$ de La Lettre de l'OCIM « Regards sur le patrimoine scientifique des universités » qui rassemble les principales contributions à ce colloque).

3. Georges Larrouy : docteur en médecine, il fut aussi président de l'université Toulouse III - Paul Sabatier, de l'Académie des Sciences, Inscriptions et Belles-Lettres de Toulouse, ainsi que du Conseil scientifique du muséum d'Histoire naturelle de Toulouse.

4. L'IDEX, pour Initiatives d'excellence, fait partie des "investissements d'avenir" ou "grand emprunt", programme d'investissement de l'État français dont le but est de créer en France des ensembles pluridisciplinaires d'enseignement supérieur et de recherche de rang international et dont l'université de Toulouse a bénéficié entre 2012 et 2016.

5. Loi $n^{\circ}$ 2013-660 sur l'enseignement supérieur et la recherche adoptée le 22 juillet 2013 organisant le regroupement des établissements universitaires. Les statuts de l'UFTMiP ont été votés en juillet 2014 .

6. Jolivet, A.-C. et Mazens, M.-C. Le patrimoine scientifique des universités, une construction à la frontière de plusieurs mondes, in Des patrimoines et des normes (formation, pratique et perspectives). Collection du CTHDIP, Études d'histoire du droit et des idées politiques, n² 21, 2015, pp. 67-76.

7. université Toulouse 1 Capitole (UT1), université Toulouse Jean-Jaurès (UT2), université Toulouse III - Paul Sabatier (UT3), Institut National Polytechnique de Toulouse (INP Toulouse), Institut National des Sciences Appliquées de Toulouse (INSA) et Institut Supérieur de l'Aéronautique et de l'Espace (ISAE). Nous ne pouvions pas, en toute conscience, ignorer le patrimoine du Centre National de la Recherche Scientifique (CNRS) qui partage la tutelle de grands instituts toulousains (l'Institut de la Mécanique des Fluides de Toulouse IMFT) ou dont les laboratoires propres sont emblématiques pour la région (le Centre d'Élaboration de Matériaux et d'Études Structurales CEMES et le Laboratoire d'Analyse et d'Architecture des Systèmes LAAS).

8. Téléchargement sur www.univ-toulouse.fr/universite/actualites/le-patrimoine-scientifiquetoulousain-desormais-son-ouvrage 


\section{RÉSUMÉS}

En 2014 et 2015, l'université Fédérale Toulouse Midi-Pyrénées a mené une étude sur l'ensemble des fonds et collections du site, sur le patrimoine scientifique mais aussi bâti, artistique, écrit et archivistique : l'enjeu était d'établir une méthodologie puis de créer un rapport qui rende compte de la richesse intrinsèque des héritages mais aussi des mécanismes de prises en charge à l'œuvre et de l'implication des acteurs de la communauté universitaire ou des partenaires institutionnels.

\section{AUTEURS}

\section{ANNE-CLAIRE JOLIVET}

chef de projet PATSTEC (PATrimoine Scientifique et TEchnique Contemporain) de Midi-Pyrénées, au sein de l'université Fédérale Toulouse Midi-Pyrénées anne-claire.jolivet@univ-toulouse.fr

\section{MARIE-CHARLOTTE MAZENS}

chargée de l'étude du patrimoine culturel universitaire pour l'université Fédérale Toulouse MidiPyrénées) d'avril à décembre 2014, est dirigeante de l'Agence d'Ingénierie culturelle Culturalys mariecharlotte.mazens@gmail.com 